\title{
COMMENTARY
}

\section{Hypovitaminosis D and morbidity in critical illness: is there proof beyond reasonable doubt?}

\author{
Bala Venkatesh ${ }^{1,2,3^{*}}$ and Priya Nair ${ }^{4,5,6}$ \\ See related research by Amrein et al., http://ccforum.com/content/18/2/R47
}

\begin{abstract}
Vitamin $\mathrm{D}$ is recognized to have important actions outside its well-recognized role in musculoskeletal health. These include antimicrobial action, antiinflammatory, and cardio-protective properties. A high prevalence of vitamin D deficiency and its association with adverse clinical outcomes have now been widely documented in observational studies in the critically ill. These studies of association, however, do not necessarily imply causation, as vitamin D deficiency may be merely a marker of higher illness severity and consequently poorer outcomes. This issue can be clarified only by undertaking high-quality randomized controlled trials of vitamin D supplementation in this vulnerable population.
\end{abstract}

\section{Introduction}

In the previous issue of Critical Care, Amrein and colleagues [1] report a single-center observational study of 655 surgical and non-surgical critically ill patients over a 2-year period and describe the association between vitamin D status and seasonality and mortality in critical illness. Vitamin D, traditionally considered important for the maintenance of calcium homeostasis and musculoskeletal health, is now widely recognized for its extraskeletal 'pleiotropic effects'. Over the past decade, data from biochemical and molecular genetic studies indicate that vitamin D - particularly its active form, 1,25-dihydroxy cholecalciferol (1,25-dihydroxy-D3) - has a much wider role. These pleiotropic effects include potentiation of antimicrobial action and cardio-protective and immunomodulatory effects. The discoveries that most tissues

\footnotetext{
* Correspondence: bala.venkatesh@health.qld.gov.au

${ }^{1}$ Princess Alexandra Hospital, 199 Ipswich Road, Wooloongabba QLD 4102, Australia

${ }^{2}$ Intensive Care, The Wesley Hospital, 451 Coronation Drive, Auchenflower QLD 4066, Australia

Full list of author information is available at the end of the article
}

and cells in the body have vitamin D receptors and that several possess the 1- $\alpha$ hydroxylase enzyme have provided novel insights into the pleiotropic actions of this vitamin [2].

\section{Hypovitaminosis D: Prevalence and associations with clinical outcome}

Vitamin D is synthesized in the skin and then undergoes 25-hydroxylation in the liver to 25-hydroxy-D3 followed by $1 \alpha$-hydroxylation, especially but not exclusively in the kidneys, to 1,25-dihydroxy-D3. Based on optimal bone health, a widely used definition considers a serum 25hydroxy-D3 of less than $20 \mathrm{ng} / \mathrm{mL}(50 \mathrm{nmol} / \mathrm{L})$ to be a vitamin D deficiency, 20 to $30 \mathrm{ng} / \mathrm{mL}$ ( 50 to $75 \mathrm{nmol} / \mathrm{L}$ ) to be insufficient, and more than $30 \mathrm{ng} / \mathrm{mL}(75 \mathrm{nmol} / \mathrm{L})$ to be normal. Although renal $1 \alpha$-hydroxylase activation may be important for circulating 1,25-dihydroxy-D3 levels, locally 1,25 -dihydroxy-D3 formed by tissue $1 \alpha-$ hydroxylase is critical in mediating the pleiotropic actions of vitamin D. Vitamin D sufficiency is required for both the endocrine and paracrine arms of the axis to function effectively.

There is increasing evidence that vitamin D deficiency is common in hospitalized patients and particularly in the critically ill. Hypovitaminosis D has been associated with increased incidence of a number of autoimmune conditions and adverse outcomes in cardiac transplant recipients and the general population [3]. Although vitamin D insufficiency does not pose immediate health hazards in otherwise healthy individuals, it may be detrimental during critical illness, as the circulating vitamin D pool functions as an important reservoir for local conversion to the active metabolites at tissue level during stress [4].

Global interest in the significance of vitamin D deficiency in critical illness was triggered in 2009 following a report by Lee and colleagues [5], who noted a previously under-recognized high prevalence of hypovitaminosis D. Only $7 \%$ of patients were vitamin D-sufficient, and a predicted mortality was three times higher in 
deficient patients. Subsequently, a number of studies in critically ill patients have reported a prevalence ranging from $38 \%$ to $100 \%$, which is about $50 \%$ higher than that for patients in general medical wards.

Several observational studies in critically ill patients have also demonstrated an association between vitamin D deficiency/insufficiency and poor outcomes. These include increases in illness severity and risk of death, ICU length of stay [6-8], ventilator requirement [9], rates of infection (including ventilator-associated pneumonia) [10], blood culture positivity [11], organ dysfunction, and short-term and long-term hospital mortality [5,9,11]. Furthermore, ICU and hospital costs were found to be higher for vitamin D-deficient patients.

In this context, the study findings of Amrein and colleagues are in accord with previously published data. Vitamin D levels were categorized by month-specific tertiles (high, intermediate, and low) to reflect seasonal variation of serum 25-hydroxy-D3 levels. There was a high prevalence of vitamin D deficiency and insufficiency. Adjusted hospital mortality was significantly higher in patients in the low and intermediate compared with those in the high tertiles. No sepsis-related fatality was observed in the highest month-specific tertile or in vitamin D sufficiency. The authors concluded that low 25-hydroxy-D3 status is significantly associated with all-cause and sepsis mortality in the critically ill and suggested that interventional studies are warranted to study the effect of vitamin D supplementation on outcome in this population. However, the indications for vitamin $\mathrm{D}$ assays in these patients were unclear; it was a convenience sample resulting in a selection bias as only patients who had vitamin D assays performed were chosen. There were no differences in bacteremia between survivors and non-survivors. Data on inflammatory markers were not reported. Moreover, vitamin D levels are influenced by fluid shifts and diurnal variability, throwing into doubt the significance of a single measurement $[12,13]$.

Despite these limitations, the study adds to the burgeoning volume of literature on the prevalence of hypovitaminosis $\mathrm{D}$ in critically ill patients and the resulting potential for adverse outcomes. Where do we go from here? There have been calls for universal vitamin D supplementation in critically ill patients. Given the present evidence, this would be premature as a number of caveats still remain.

The criteria for definitions of vitamin D sufficiency are based on bone health; the optimal level for pleiotropy remains unclear. Moreover, little data exist on the bioactive form, 1,25-dihydroxy-D3, in critical illness and association with outcomes. Reports of supra-physiological levels of 1,25-dihydroxy-D3 following inflammatory response in critically ill patients [12] and, more recently, evidence of extra-renal production of 1,25-dihydroxy-D3
[14] have been published. Furthermore, the high degree of protein binding of 25-hydroxy-D3 and consequently the relevance of free levels, particularly in the context of altered pharmacokinetics associated with critical illness, remain to be elucidated.

\section{Conclusions}

The observational studies available do not suggest causation, and hypovitaminosis D may be simply a marker of poor health, which predicts patients who would have poorer outcomes. Reports that supra-physiological levels of 25-hydroxy-D3 may be associated with adverse outcomes are also emerging [15]. The only means to clarify the role for supplementation is to conduct appropriate dose-response studies followed by a high-quality placebocontrolled randomized trial of vitamin $\mathrm{D}$ supplementation in this vulnerable population. Vitamin D is inexpensive and, if it is shown to be beneficial, there is no barrier to rapid implementation into routine clinical practice worldwide.

\section{Competing interests}

The authors declare that they have no competing interests.

\section{Author details}

'Princess Alexandra Hospital, 199 Ipswich Road, Wooloongabba QLD 4102, Australia. ${ }^{2}$ Intensive Care, The Wesley Hospital, 451 Coronation Drive, Auchenflower QLD 4066, Australia. ${ }^{3}$ University of Queensland, Brisbane, St Lucia QLD 4072, Australia. 'Intensive Care Unit, St Vincent's Hospital, 41 Victoria Parade, Fitzroy VIC 3065, Australia. ${ }^{5}$ University of New South Wales, High Street, Kensington NSW 2052, Australia. ${ }^{6}$ Garvan Institute of Medical Research \& George Institute for Global Health, 384 Victoria Street,

Darlinghurst Sydney NSW 2010, Australia.

Published: 08 May 2014

\section{References}

1. Amrein K, Zajic P, Schnedl C, Waltensdorfer A, Fruhwald S, Holl A, Urbanic Purkart T, Wünsch G, Valentin T, Grisold A, Stojakovic T, Amrein S, Pieber TR, Dobnig H: Vitamin D status and its association with season, hospital and sepsis mortality in critical illness. Crit Care 2014, 18:R47.

2. Bikle D: Non-classic actions of vitamin D. J Clin Endocrinol Metab 2009, 94:26-34.

3. Thacher TD, Clarke BL: Vitamin D insufficiency. Mayo Clin Proc 2011, 86:50-60.

4. Lee $P$, Nair $P$, Eisman JA, Center JR: Vitamin D deficiency in the intensive care unit: an invisible accomplice to morbidity and mortality? Intensive Care Med 2009, 35:2028-2031.

5. Lee P, Eisman JA, Center JR: Vitamin D deficiency in critically ill patients. N Eng J Med 2009, 360:1912-1913.

6. Matthews LR, Ahmed Y, Wilson KL, Griggs DD, Danner OK: Worsening severity of vitamin $D$ deficiency is associated with increased length of stay, surgical intensive care unit cost, and mortality rate in surgical intensive care unit patients. Am J Surg 2012, 204:37-43.

7. McKinney JD, Bailey BA, Garrett LH, Peiris P, Manning T, Peiris AN: Relationship between vitamin D status and ICU outcomes in veterans. Am Med Dir Assoc 2011, 12:208-211.

8. Nair P, Lee P, Reynolds C, Nguyen ND, Myburgh J, Eisman JA, Center JR: Significant perturbation of vitamin D-parathyroid-calcium axis and adverse clinical outcomes in critically ill patients. Intensive Care Med 2013, 39:267-274.

9. Venkatram S, Chilimuri S, Adrish M, Salako A, Patel M, Diaz-Fuentes G: Vitamin D deficiency is associated with mortality in the medical intensive care unit. Crit Care 2011, 15:R292. 
10. Flynn L, Zimmerman LH, McNorton K, Dolman M, Tyburski J, Baylor A, Wilson R, Dolman H: Effects of vitamin D deficiency on critically ill surgical patients. Am J Surg 2012, 203:379-382.

11. Braun AB, Gibbons FK, Litonjua AA, Giovannucci E, Christopher KB: Low serum 25-hydroxyvitamin $D$ at critical care initiation is associated with increased mortality. Crit Care Med 2012, 40:63-72.

12. Krishnan A, Ochola J, Mundy J, Jones M, Kruger P, Duncan E, Venkatesh B: Acute fluid shifts influence the assessment of serum vitamin $D$ status in critically ill patients. Crit Care 2010, 14:R216.

13. Venkatesh B, Davidson B, Robinson K, Pascoe R, Appleton C, Jones M: Do random estimates of vitamin $D$ and parathyroid hormone reflect the 24-hour profile in the critically ill? Intensive Care Med 2012, 38:177-179.

14. Krishnan A, Grosso A, Dimeski G, Grice J, Jones M, Venkatesh B: Evidence for extra-renal production of $1 a, 25(\mathrm{OH})_{2} \mathrm{D}_{3}$ in critical illness: a preliminary study. Intensive Care Med 2013, 39:1505-1506.

15. Ralph R, Peter JV, Chrisphal A, Zachariah A, Dian J, Sebastian T, Venkatesh B, Thomas K: Supraphysiological 25-hydroxy vitamin D3 level at admission is associated with illness severity and mortality in critically ill patients. J Bone Miner Metab. in press.

$10.1186 / \operatorname{cc} 13863$

Cite this article as: Venkatesh and Nair: Hypovitaminosis D and morbidity in critical illness: is there proof beyond reasonable doubt? Critical Care 2014, 18:138 